\title{
Dos son mejor que uno
}

\author{
Two are better than one
}

\author{
Antonio Anzueto* \\ *Pulmonary and Critical Care Medicine, South Texas Veterans Health Care System. \\ University of Texas Health Science Center at San Antonio.
}

\begin{abstract}
Dos son mejor que uno porque tienen mejor paga por su trabajo. Porque si cayeren, el uno levantará a su compañero, pero iAy del solo! que cuando cayere, no habrá segundo que lo levante. Eclesiastés 4:9-12
\end{abstract}

La medicina crítica es una de las disciplinas más recientes en medicina. En 1952, durante la epidemia de polio en Copenhague, Dinamarca, el anestesiólogo Dr. Bjorn Ibsen describió en estos pacientes cómo un manejo integral de la vía aérea en combinación con ventilación mecánica positiva, junto con todos los demás aspectos médicos, resultó en una notable disminución en mortalidad. Ésta fue la primera descripción en épocas recientes de lo que en el futuro se convertiría en el cuidado crítico de los enfermos. Posteriormente, los anestesiólogos lideraron la creación de unidades de cuidado intensivo enfocadas en el manejo ventilatorio del paciente posoperado. A medida que la tecnología avanzó durante los años 1960 y 1970, se fue formalizando la creación de estas unidades. ${ }^{2}$ Estas tecnologías incluyeron el monitoreo cardiopulmonar no invasivo y disponibilidad de ventiladores que con un aumento en el conocimiento de la fisiopatología del paciente crítico empezó a atraer a otros grupos médicos al campo del cuidado crítico. ${ }^{3}$ En los años 1980 The American Board of Medical Specialties (ABMS, por sus siglas en inglés) empezó el proceso de reconocer el entrenamiento en medicina crítica en los Estados Unidos. Al mismo tiempo, la medicina pulmonar se fue reinventando, inicialmente médicos interesados en condiciones como la tuberculosis fueron los primeros que

Correspondencia:

Antonio Anzueto

Correo electrónico: anzueto@uthscsa.edu

Trabajo recibido: 31-VII-2020; aceptado: 04-VIII-2020.

Citar como: Anzueto A. Dos son mejor que uno. Neumol Cir Torax. 2020;79(3):132-133. https://dx.doi.org/10.35366/96645 se separaron del área de medicina interna para convertirse en especialistas. Así se crearon centros especializados en el manejo del paciente con patología pulmonar como los sanatorios antituberculosos. La neumología ha continuado desarrollándose en otras áreas como función pulmonar, broncoscopia, sueño, etc. Esta transición en la neumología ha ocurrido en todo el mundo.

Estados Unidos (EE.UU.) ha sido uno de los pocos países donde se integró la neumología clínica y la medicina crítica en los años 80. La Sociedad Americana del Tórax (ATS, por sus siglas en inglés) reconoció la importancia de la combinación entre enfermedades pulmonares y cuidado crítico, creando la Asamblea de Cuidado Crítico, y en 1994 la revista de la ATS incorporó «cuidado crítico» en el título. ${ }^{4}$ Asimismo, se crearon los programas de entrenamiento acreditados por el Program Requirements for Graduate Medical Education (ACGME, por sus siglas en inglés) donde se combina la formación de medicina pulmonar con cuidado crítico. ${ }^{5}$ Actualmente, en EE.UU. más de $98 \%$ de los programas de neumología son programas combinados con cuidado crítico. Los médicos después de graduarse completan tres años de entrenamiento en medicina interna, luego dos años en neumología y un año en cuidado crítico. ${ }^{6}$

La integración del programa de enfermedades pulmonares y cuidado crítico como existe en EE.UU. se ha venido desarrollando en varias regiones del mundo. En 2014, Qiao y colaboradores describieron en China la necesidad de crear un proceso para la formación de médicos en el área de enfermedades pulmonares y cuidado crítico para poder servir a una población que está aumentando y al mismo tiempo va envejeciendo. ${ }^{7}$ Como resultado se estableció una colaboración entre la Chinese Thoracic Society (CTS) y el American College of Chest Physicians (CHEST) para desarrollar los requerimientos y la currícula necesaria para poder establecer un programa de posgrado que incluyera un entrenamiento en estas áreas. ${ }^{8}$

En los últimos 20 años, la insuficiencia respiratoria aguda asociada con procesos infecciosos pulmonares como la neu- 
monía adquirida en la comunidad, tanto bacterianas como virales, recientemente la pandemia por influenza A H1N1 en 2009 y en la actualidad la pandemia de COVID-19 por el virus SARS-CoV-2 ha creado la necesidad de integrar neumología y cuidado crítico para el manejo de pacientes agudos.

Por este medio felicito al Dr. Juan Carlos Vázquez García y coautores por publicar una propuesta de integración de neumología y cuidado crítico en el Instituto Nacional de Enfermedades Respiratorias Ismael Cosío Villegas (INER) en la Ciudad de México. ${ }^{9}$ El INER durante las pandemias recientes se ha convertido en un centro nacional de referencia para el manejo de estos pacientes. De hecho, es una institución que fue creada para el manejo de pacientes con tuberculosis, y actualmente se ha convertido en un centro de excelencia para el manejo de patologías respiratorias agudas y crónicas desde asma hasta enfermedades intersticiales. También es un centro de referencia de pacientes con infecciones pulmonares. El INER es la institución ideal donde se puede implementar un programa combinado de enfermedades pulmonares y cuidado crítico, ofreciendo a los residentes una gran oportunidad de desarrollar su carrera profesional. Yo creo que estos programas deben tener el aval de instituciones educativas así como de organizaciones profesionales.

Actualmente, también se ha reconocido que hay un aumento en la demanda de médicos en cuidado crítico en todo el mundo, y la cantidad disponible no es suficiente para cubrir la demanda. ${ }^{10,11}$ Asimismo, la mayoría de los médicos que se entrenan en el INER se van a trabajar en los estados del país, donde hay un número muy limitado de intensivistas y neumólogos. Los médicos entrenados tanto en enfermedades pulmonares como en cuidado crítico podrán ofrecer mayor apoyo a la comunidad. Dadas las crisis mundiales que estamos viviendo, es imperativo poder aumentar la cantidad de médicos que tienen entrenamiento en diferentes áreas de la medicina, siendo el cuidado crítico una de las más importantes.

\section{REFERENCIAS}

1. Colice GL. Historical perspective on the development of mechanical ventilation. In: Tobin MJ, editor. Principles and practice of mechanical ventilation. New York: McGraw-Hill; 1994. 1-35.
2. Safar $P$, Grenvik A. Organization and physician education in critical care medicine. Anesthesiology. 1977;47(2):82-95. Available in: https:// doi.org/10.1097/00000542-197708000-00002

3. Tobin MJ, Grenvik A. Critical care medicine, whither from and whither to. Ir Med J. 1983;76(11):462-463.

4. Wilson KC, Corn J. AJRCCM: 100-year anniversary. The History of Official American Thoracic Society Documents. Am J Respir Cri Care Med. 2017;195(9):1115-1117. Available in: https://doi.org/10.1164/ rccm.201703-0527ED

5. Brotherton SE, Etzel SI. Graduate medical education, 2014-2015. JAMA. 2015;314(22):2436-2454. Available in: https://doi.org/10.1001/ jama.2015.10473

6. ACGME Program Requirements for Graduate Medical Education in Pulmonary and Critical Care Medicine (Internal Medicine). Available in: https://www.acgme.org/Portals/0/PFAssets/ProgramRequirements/156_ pulmonary_critical_care_int_med_2020.pdf.

7. Qiao R, Rosen MJ, Chen R, Wu S, Marciniuk D, Wang C; CTS-ACCP Pulmonary and Critical Care Medicine Workgroup. Establishing pulmonary and critical care medicine as a subspecialty in China: joint statement of the Chinese thoracic society and the American college of chest physicians. Chest. 2014;145(1):27-29. Available in: https:// doi.org/10.1378/chest.13-2082

8. Qiao R, Marciniuk D, Augustyn N, Rosen MJ, Dai H, Chen R, et al.; China-CHEST PCCM Program Steering Committee. Establishing pulmonary and critical care medicine in China: 2016 report on implementation and government recognition: Joint Statement of the Chinese Association of Chest Physicians and the American College of Chest Physicians. Chest. 2016;150(2):279-282. Available in: https:// doi.org/10.1016/j.chest.2016.05.005

9. Vázquez-García JC, Salas-Hernández J, Pérez-Padilla R, BáezSaldaña AR, Vega-Barrientos RS, Carrillo-Alduenda JL, et al. Neumología y medicina crítica como una especialidad integrada. La respuesta obligada en la era de las pandemias respiratorias. Neumol Cir Torax. 2020;79(3):134-140.

10. Kelley MA, Angus D, Chalfin DB, Crandall ED, Ingbar D, Johanson $W$, et al. The critical care crisis in the United States: a report from the profession. Chest. 2004;125(4):1514-1517. Available in: https://doi. org/10.1378/chest.125.4.1514

11. Kahn JM, Rubenfeld GD. The myth of the workforce crisis. Why the United States does not need more intensivist physicians. Am J Respir Crit Care Med. 2015;191(2):128-134. Available in: https://doi. org/10.1164/rccm.201408-1477cp

Conflicto de intereses: El autor declara no tener conflicto de intereses. 